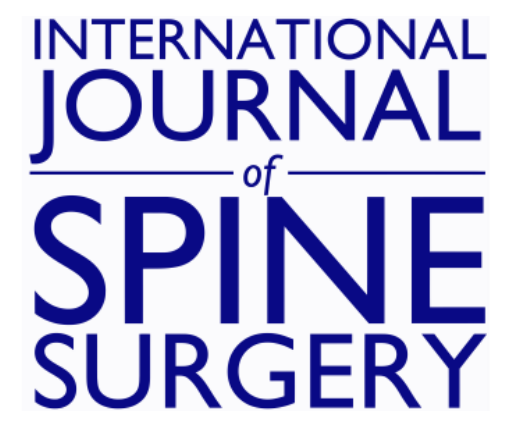

\title{
Foreword, Biomechanics Special Issue
}

Boyle Cheng and Vijay Goel

Int J Spine Surg 2015, 9 ()

doi: https://doi.org/10.14444/2031

http://ijssurgery.com/content/9/31

This information is current as of April 26, 2023.

Email Alerts Receive free email-alerts when new articles cite this article. Sign up at: http://ijssurgery.com/alerts

The International Journal of Shines Surgerht 2397 Waterbury Circle, Suite 1,

Aurora, IL 60504, Phone: +1-630-375-1432

(C) 2015 ISASS. All Rights Reserved. 


\section{Foreword, Biomechanics Special Issue}

Boyle Cheng, PhD,1,2 Vijay Goel, PhD 3

${ }^{1}$ Department of Neurosurgery, Allegheny General Hospital, Pittsburgh, PA, 2 Department of Neurosurgery, Drexel University College of Medicine, Pittsburgh, $P A,{ }^{3}$ Engineering Center for Orthopaedic Research Excellence, Departments of Bioengineering and Orthopaedic Surgery, Colleges of Engineering and Medicine, University of Toledo, Ohio

Volume 9 ARTICLE 31 - BIOMECHANICS SPECIAL ISSUE DOI: 10.14444/2031

\section{The Relevance of Clinical}

\section{Biomechanics}

Does biomechanics matter? Moreover, how do the frequently reported positive biomechanical studies results compare with the clinical outcomes? This is a question whose answer appears to change over time. Often at the outset of a new spinal technology with carefully controlled release conditions including careful patient selection through strict inclusion criteria, the clinical outcomes tend to be positive. As the technology undergoes a more widespread release, the long term outcomes often do not remain as positive as the early outcomes. Additionally, newly treated patients may not experience any of the outcomes nor subsequent benefits at the same follow up time points as reported by the study patients. Yet, throughout the products cycle, the fundamental biomechanical results are often consistent even with multiple independent biomechanical studies. With the constant release of new technologies comes a constant influx of new data, and given the plethora of overwhelming positive biomechanical results that are often reported, it is difficult to understand which results are clinically relevant if any at all. Further clarification and understanding of reported biomechanical data is necessary in order for biomechanics to remain clinically relevant.

The results of biomechanical studies require a useful working knowledge of the biomechanical study design. The assumptions intrinsic to the protocol explicitly dictate the conditions that each test specimen will be subjected. In turn, this governs the generated data and subsequent conclusions that can be drawn. This is akin to the inclusion and exclusion criteria that govern the enrolled patient population in clinical trials. Moreover, the results are specific to the study population withal the appropriate conditions. The further away the spine patient is away from the stud- ied randomized control trial (RCT) population, the more variable the results. Thus, when the results are reported for biomechanical studies, take into consideration under what conditions these results are reported.

Many metrics may be used to gage the biomechanical performance of an implant or treatment. Historical evidence of biomechanics having a meaningful clinical impact is abundant. However, there exist incidences of biomechanical results being misinterpreted. Ramifications range from having device designs that are insufficiently robust to suboptimal clinical treatments. Recent examples related to the spine include devices that are not approved by regulatory agencies and catastrophic implant failures of posterior lumbar dynamic systems in patients. Starting with basic anatomy, communication between biomechanicians and the clinical spine specialist must be as clear and easily understood as possible.

The human spine is made up of 22 vertebrae and is classified into three distinct segments - the cervical spine, the thoracic spine, and the lumbar spine. Both the cervical and lumbar spines are lordotically contoured in the sagittal plane, whereas the thoracic spine is naturally kyphotic. Spinal implant technologies and associated surgical techniques may involve single or multiple vertebrae. Under normal conditions, the individual vertebrae work in conjunction with adjacent vertebrae. The simplest grouping of vertebrae that can register relative spinal motion is referred to as the functional spinal unit (FSU). An FSU consists of two adjacent vertebrae and the associated osteoligamentous structures. Biomechanical studies involve either individual, or a collection of FSUs or even segments. Particular importance has been focused on the three segments often treated as separate structures. However, as segmental junction issues arise clinically including at the cervicothoracic and thoracolumbar junctions, revisiting biomechani- 
cal testing strategies may be warranted. Recently, the occiptocervical and lumbosacral as well as the sacroiliac joint have also become popular study topics.

Early studies comparing the effectiveness of fixation devices, e.g., rigid rod based pedicle screws compared to wired rods, have helped technologies achieve mainstream acceptance. Through the appropriate metric, often the range of motion (ROM) of the FSU, fixation technologies have been justified through biomechanical benchtop studies for validation as a clinical treatment. A reduction in ROM in biomechanical studies served as sufficient validation for clinical use when evaluating early fixation techniques. In the modern era, the value of biomechanical results increases when correlated to the clinical outcomes and since the results of the biomechanical studies are applicable to controlled and specific test conditions, a greater emphasis should be placed on the breadth of applicability. Moreover, the ability to detect differences between treatments should be a primary focus to differentiate products with the same clinically labeled indication. Methods to increase the utility of the benchtop biomechanical studies should focus on broader conditions for clinical generalizations and the necessary sensitivity to detect device specific differences. This would strengthen biomechanical considerations and allow for earlier involvement in the design phase and consequently, more integral to the overall design process.

Often, finite element modeling (FEM), in vitro benchtop testing and animal studies are included in the body of knowledge contributing to useful predictions in clinical outcomes. It is the repeatability coupled with the relative ease of use that makes FEM a meaningful part of the design process. Whether it is the results related to strain on an implant design or the gross displacement on instrumented validated FSU model, FEM provides a means to rapidly compare design iterations and the potential ramifications associated with each design on a standardized virtual model. The sophistication of these models has steadily increased as material properties associated with FSUs become more detailed and computational power increases. Further improvements in this area should consider musculature effects as well as whole body alignment.

Pure moment based test protocols ${ }^{1}$ recommended by Manohar Panjabi have become a de facto means to validate FEM FSUs and also the de facto standard in biomechanical cadaveric testing of the treated FSU when comparing to the pathologic spine. Due to the interlaboratory reliability, pure moment testing is a significant advancement from other biomechanical tests $^{2}$ such as the four point bend test and a major factor in the wide spread acceptance of flexibility protocols. However, by design and due to the lack of shear, biomechanical pure moment tests may not perfectly model physiologic bending. It remains an excellent comparative test protocol and one that can be repeated in other independent labs. As previously stated, clinical dynamic radiographic exams have been reported for clinical subjects utilizing the same ROM metric. Advancements through semiautomated computer recognition in dynamic motion analysis or improved differentials between asymptomatic and symptomatic vertebrae, clinical imaging studies have relied on biomechanical principles in assisting physicians and biomechanicians understand the similarities and differences between cadaveric benchtop tests and patient outcomes. In order to be clinically predictive for patient outcomes, the assumptions made in biomechanical pure moment tests related to the minimization of shear needs to be understood relative to the context of human subject bending.

Animal models have served as precursors to human clinical evaluations of new technologies. The physiologic response, from the cellular to the functional response in large animals, predicts some of the potential human clinical effects through the correct study design. With the appropriate models, a number of new materials including titanium alloys, surface treated metals, and coated polymers have been tested for biocompatibility in a long bone animal model. Many different models have proven suitable for such biocompatibility purposes. Functional models that incorporate both the bone-implant interface response as well as implanted functional responses of the FSU have also been used. In the cervical spine, ovine and caprine animal models have validated cervical technologies including cervical total disc replacements. 
For lumbar models, porcine models are prevalent and non-human primate models are considered. In functional testing of these devices, it is important to evaluate not only the bone implant interface over an extended period of time but it also affords insight into potentially catastrophic failure modes of the new technologies.

The use of appropriate biomechanical metrics should be applied to three distinct clinically relevant areas. The future of the field should significantly contribute to the advancement of spinal diagnostics, new implant technology innovation and evaluation of clinical effectiveness. In many ways the diagnosis and functional outcomes rely on the same radiographic parameters. As such, biomechanical considerations and technological advancements may continue to contribute not only in the assessment of normal and pathologic conditions but also the outcomes immediately post operatively and at numerous clinical milestones. These considerations depend on the static geometrical relationships of the anatomy, e.g. sagittal balance, pelvic incidence, or sacral slope. When motion is expressed, both clinically relevant and benchtop cadaveric tests report results in a planar two dimensional metric, most often ROM. Future advances, particularly related to detecting differences between new technologies, will require metrics to supplement ROM.

The importance of predicting device failure in benchtop in vitro biomechanical studies must also be given sufficient emphasis. Biomechanical research has advanced to the point where failure of devices should be detected prior to the implantation in patients. Devices benefit patients from biomechanical characteristics that perform as the design was intended. For example, fixation devices need to limit motion and stiffen the FSU after implantation. These results are definable in cadaveric testing and similar results can be found in animal studies. ${ }^{3}$ Related to fusion devices, once arthrodesis is achieved, the implant becomes obsolete. For motion preservation devices including total disc replacements the endpoint may be different. The device is expected to perform in conjunction with the remaining osteoligamentous structures for potentially a much longer period of time. As such, the biomechanical performance of motion preservation devices behaves differently than that of fixation devices and requires accurate biomechanical characterization. The ability to detect statistically significant differences between the intact, pathologic and treated conditions has been well established in the published literature for fixation devices. Detecting these differences between motion preservation devices has not been as straightforward and may require additional acceptance criteria along with the appropriate metrics for evaluation. For newer technologies, one criterion is still to provide stability while additional criterion, depending on the design intent, is to perform in a specific manner. Currently, these acceptance criteria are not well defined. Test protocols that incorporate multi modal testing and or combined mode testing have been and should be further explored. Metrics such as interpedicular travel (IPT) or interpedicular displacement (ID), have been suggested and are necessary to accurately characterize the performance of a device, particularly newer technologies found in motion preservation devices. $^{4}$

Clearly, spinal technologies have benefitted and will continue to benefit from clinically relevant biomechanics. Early incorporation of biomechanical studies with the appropriate metrics should provide guidance on the efficacy of technology for physician triage in the clinical management of spinal patients. As failed technologies for spinal treatment, particularly related to lumbar implant designs, further consideration should be given for meaningful preclinical data. The studies and techniques that are included in this issue are examples of current clinically relevant biomechanical studies.

\section{References}

1. Panjabi MM. The stabilizing system of the spine. Part I. Function, dysfunction, adaptation, and enhancement. J Spinal Disord 1992;5:383-9; discussion 97.

2. Goel VK, Panjabi MM, Patwardhan AG, et al. Test protocols for evaluation of spinal implants. Journal of Bone and Joint Surgery American edition 2006;88 Suppl 2:103-9.

3. Wilke H-J, Kettler A, Claes LE. Are Sheep

Downloaded from http://ijssurgery.com/ by guest on April 26, 2023 
Spines a Valid Biomechanical Model for Human Spines? Spine 1997;22:2365-74.

4. Cook DJ, Yeager MS, Cheng BC. Interpedicular Travel in the Evaluation of Spinal Implants: An Application in Posterior Dynamic Stabilization.

Spine 2012;37:923-31.
Published 17 July 2015.

This manuscript is generously published free of charge by ISASS, the International Society for the Advancement of Spine Surgery. Copyright ๑ 2015 ISASS. To see more or order reprints or permissions, see http://ijssurgery.com. 